\title{
Gravity Can be Part of the Transporting Energy by Constructing the Road into a Bio Quantum Path Design
}

\author{
Yi Yu Lai \\ Medical Center, B343, Medical Science Building, Tsinghua University, Beijing, 100084. PR China \\ Innoen non-profit organization, Mississauga, L4Z 3E4, ON, Canada \\ Email: yylai[at]innoen.org
}

\begin{abstract}
The bio quantum path has been experimented and suggested as a general model that covers both the microcosmic and macrocosmic world for surface tension regions [1]. The application of it in the macrocosmic world possibly has a longer history than that the existence of modern science, albeit the theory only presents recently. Considering this pattern in road design can save energy.
\end{abstract}

Keywords: bio quantum path, gravitational binding, surface tension region, Einstein's curved space

\section{Introduction}

Ancient Egyptian pyramid construction techniques are the controversial subject of many hypotheses. Most hypotheses agree that huge stones were carved from quarries with copper chisels, and these blocks were then dragged and lifted into position. The Twelfth Dynasty tomb of Djehutihotep has an illustration of 172 men pulling an alabaster statue of him on a sledge. The statue is estimated to weigh 60 tons, and it is estimated that 45 workers would be required to start moving a $16,300 \mathrm{~kg}$ lubricated block or eight workers to move a 2,750 kg block [2]. Parry [3] has also suggested a method for rolling the stones, using a cradle-like machine that had been excavated in various new kingdom temples. Four of those objects could be fitted around a block so it could be rolled easily. Experiments done by the Obayashi Corporation, with concrete blocks 0.8 meters square by 1.6 meters long and weighing 2.5 tons, showed how 18 men could drag the block over a 1-in-4 incline ramp, at a rate of 18 meters per minute. It is still not known whether the Egyptians took this method, but the experiments indicate it could have worked using stones of this size. Egyptologists generally accept this for the 2.5-ton blocks mostly used but do not agree over the methods used for the $15+$ ton and several 70 to 80 -ton blocks.

No matter uses the sledge, rolling device, or directly drag with some lubricants such as water, sand, ice, etc., all such friction-reducing technologies failed to take gravity to counteract friction. If the transportation road is constructed by a bio quantum path pattern, the energy or labor force could be saved due to the participation of gravity. Following the (Fig.4) and the experiment in the paper [1], human-exerting energy only needs to overcome the friction in a short distance before each peak of a bio quantum path road, on the remaining path, the friction will be spliced out by gravity. In quarries or mountain areas, establishing a road with the bio quantum path pattern follow the available terrain conditions could save much constructing labor forces. In practice, ancient Egyptian possibly had already found this way not only saved greatly in road constructions but also possessed enough transporting efficiency for moving objects on it. They could release a massive object at each peak and use a wedge to prevent the pullback at the near-next-peak region, later only need to move the object for a short distance can go to the next cycle, working in such a way could also increase the delightfulness and reduce the tediousness of the huge block moving job. In that era and even modern times, practices would always be easier to discover something new than only do the theoretical analysis; this fact gives much weight to the possibility. Nevertheless, this is yet another archaeological hypothesis that can pass the modern experimental simulations and wait for further evidence.

Today, our vehicle driving or other transportation roads can equally procure the gravity potential energy by constructing them into a bio quantum path pattern. The energy-saving effect of this design is $100 \%$ guaranteed for all the vehicles on it, just need to consider certain adapting conditions, such as some people not accustomed to driving on a wave-shaped road, the arc length and the constructing materials, the stations and intersections better on a peak region, and the bio quantum path design can save energy only for vehicles and not for pedestrians, etc. Albeit not every road or the whole range of a certain road fit for the bio quantum path pattern, the future established roads could segmentally consider this design based on available local conditions. (The highway is possibly a concrete example which quite fits for gravity potential energy-saving pattern if available terrain conditions sustaining the design. With proper arc shape design based on the bio quantum path pattern, the highway can also reduce the tediousness for long-time drivers. A long bridge also fits this design.) Nowadays, no modern road has been constructed in this model before means modern human engineering still fails to know how to make use of the gravity potential energy in vehicle transportation; life evolution does know how to utilize the gravitational energy for diverse functions. DNA palindromes are bio quantum paths; life physically integrates them with coded genes quite well. Different from the human road constructions that can only utilize the vertical gravity potential energy for energy-saving purposes, life structures can make use of the in vivo gravitational binding[1] from every direction for splicing and achieve much more bio-functions. It is a reminder that not everything of the environment we are living in follows Euclidean geometry; gravity does make the shortest distance between two points with the equivalent levels of energy-consuming and frictions

\section{Volume 9 Issue 2, February 2020




\section{International Journal of Science and Research (IJSR) \\ ISSN: 2319-7064}

ResearchGate Impact Factor (2018): 0.28 | SJIF (2018): 7.426

shakes into a bio quantum path pattern. This fact follows the concept of Einstein's curved space quite well. Equivalently, the distance between genes is no longer the number of linear nucleotide base pairs based on the Euclidean geometry due to the functions of in vivo gravitational binding [1].

\section{Competing Financial Interests}

The authors declare no competing financial interests.

\section{References}

[1] Lai, Y. Y. Bio-inertia resonates life into evolution. IJSR, 8, 1680-1718 (2019). DOI: 10.21275/ART20203017

[2] Stocks, D. A. Experiments in Egyptian Archaeology Routledge. ISBN 978-0-415-30664-5 pp.196-197 (2003).

[3] Parry, D. "Engineering the Pyramids" The History Press 2013 ISBN 978-0-750-93415-2.

Volume 9 Issue 2, February 2020

www.ijsr.net 